\title{
The Influence of Detector Quality and Throughput for Cryo-EM of Challenging Proteins
}

Joshua Mendez and Scott Stagg

Florida State University, Tallahassee, Florida, United States

Since their introduction, direct electron detectors (DEDs) have been rapidly adopted by the cryogenic electron microscopy (cryo-EM) community. The improved signal-to-noise ratio (SNR) and high DQE values across all frequencies provide a significant advantage over previous generations of detectors, making DEDs attractive alternatives [1]. With this advantage, the resolution of cryo-EM structures have been advanced and now have reached better than $2 \AA$ using single particle analysis (SPA) [2]. In the meantime, the number of new cryo-EM structures published also increased, but there remain challenging structures, especially those with sizes of $100 \mathrm{kDa}$ or lower. In 1995, it was theorized that structures of proteins with sizes as low as $38 \mathrm{kDa}$ could be resolved to $3 \AA$ [3]. But in recent years, only a few structures smaller than $100 \mathrm{kDa}$ have been determined to high resolution (sub $3 \AA$ ) [4], [5]. Size is a considerable limiting factor because poorly represented low spatial frequencies in micrographs make it challenging to assign correct Euler angles to these proteins. As a result, the accuracy and precision for alignment of cryoEM specimens decreases with decreasing particle size. For this reason, detectors with high detective quantum efficiency (DQE) at low spatial frequency are desired.

We have previously demonstrated that the DE64 detector, from Direct Electron, has a high DQE in low spatial frequencies when operated in counting mode [6]. This prompted us to investigate whether the DE64 can determine the structure of proteins whose sizes are approaching or are lower than $100 \mathrm{kDa}$. For the present study, we have chosen three different targets that vary in size and shape. We first targeted the 443 $\mathrm{kDa}$ protein complex apoferritin. Although the size of apoferritin is not approaching the lower size limit, it has been shown that it can only be resolved using high quality micrographs [7]-[9]. It also serves as a reference point from which the performance of detectors can be assessed. For the second target, aldolase was selected because of its size of $160 \mathrm{kDa}$ and D2 symmetry makes it challenging to resolve with high resolution. As for the third target, hemoglobin with a size of $64 \mathrm{kDa}$ was chosen. It has been demonstrated that hemoglobin can be resolved to $3.2 \AA$ with the help of a Volta phase plate (VPP) [4]. It was suggested that the boost in SNR provided by a VPP is necessary to resolve the structures of proteins of comparable size, but in recent years, it has been shown that SPA is capable of resolving a $64 \mathrm{kDa}$ protein without additional phase shift [5]. In addition, the atomic model of all three structures are available for validation of our results.

The biggest drawback that the DE64 faces is its slow throughput. The need for collecting large amounts of data can be reduced with high quality micrographs, although throughput is still of importance. To contrast throughput versus DQE quality, we will present each resolved structure in conjunction with a second structure generated from data collected using a K3 detector from Gatan. The K3 detector is the cryo-EM field's dominant detector with a data collection rate 1.7-fold faster than the DE64. Figure 1 shows a representative section of an aldolase micrograph collected with DE64 and its CTF fitted. In contrast Figure 2, shows a representative section of an aldolase micrograph collected with K3 and its CTF fitted. 

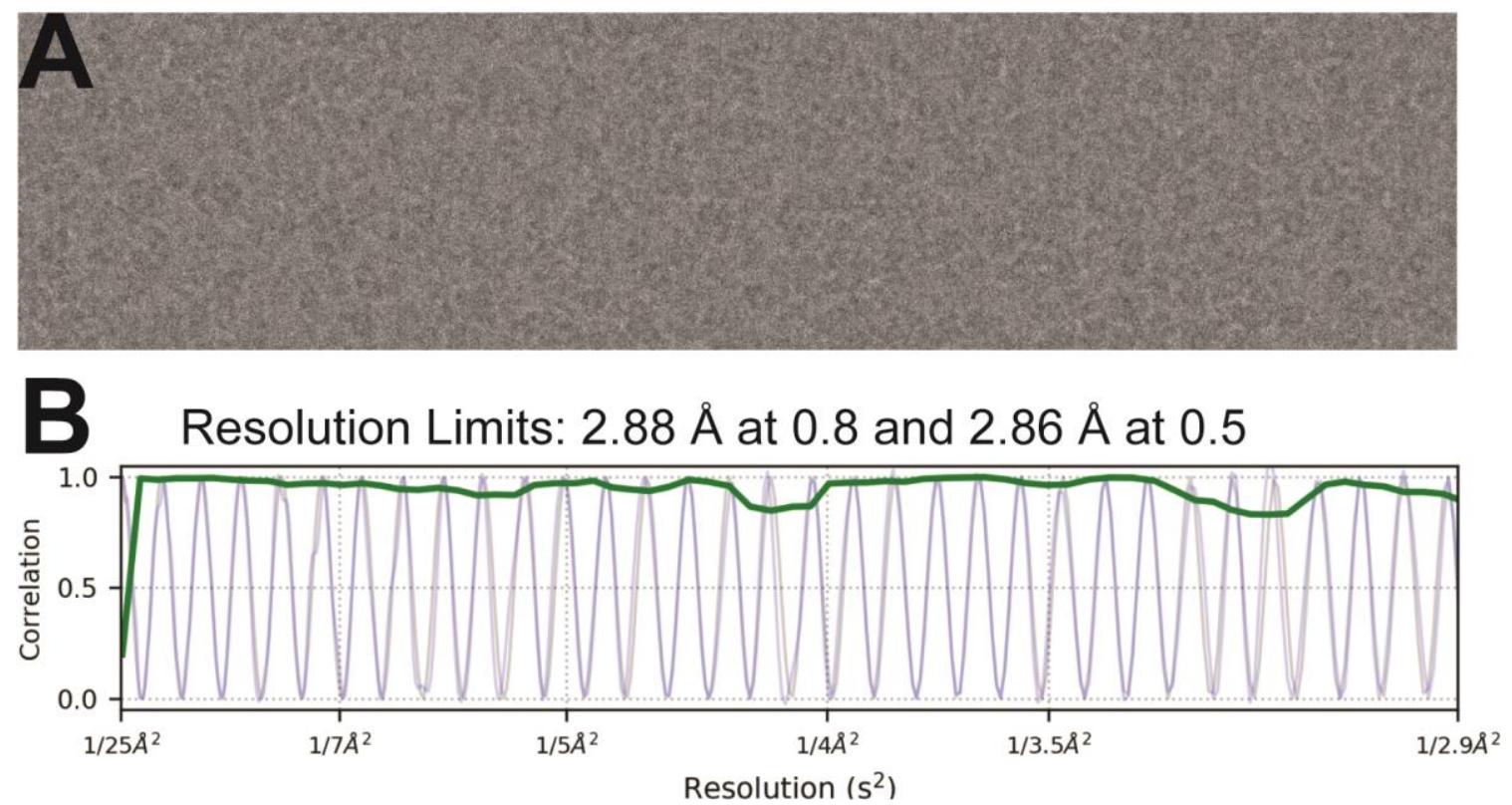

Figure 1. A) A representative section of an aldolase micrograph collected with DE64. B) The micrograph's CTF fitted to $2.88 \AA$ with a confidence value of 0.8 .

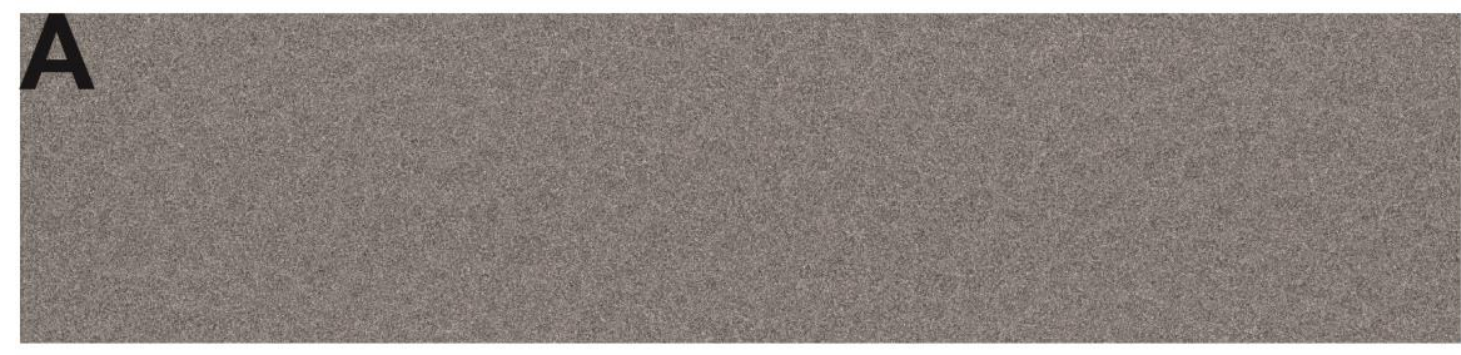

\section{B Resolution Limits: $3.16 \AA$ at 0.8 and $3.10 \AA$ at 0.5}

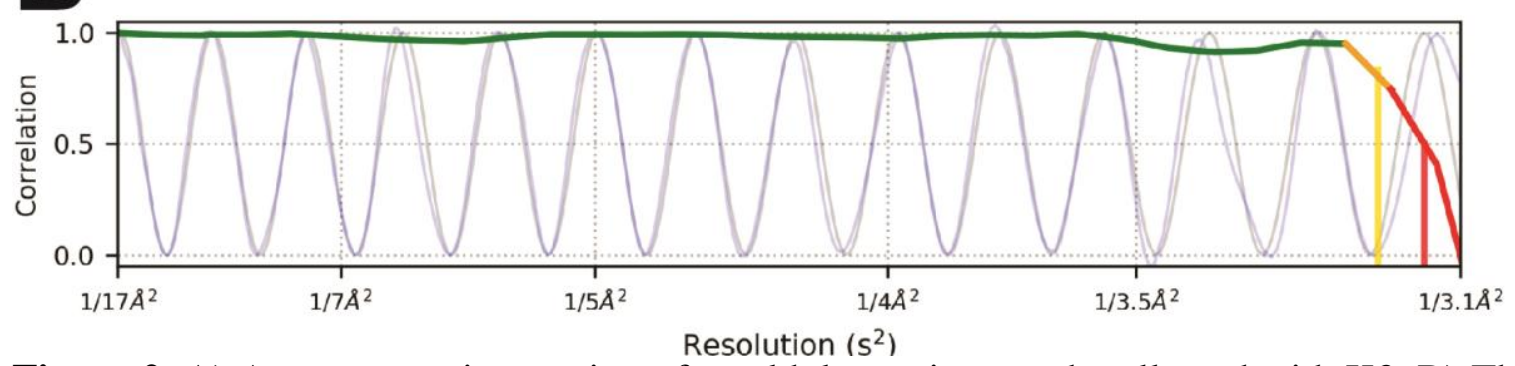

Figure 2. A) A representative section of an aldolase micrograph collected with K3. B) The micrograph's CTF fitted to $3.16 \AA$ with a confidence value of 0.8 .

\section{References}

[1] G. McMullan, S. Chen, R. Henderson, and A. R. Faruqi, "Detective quantum efficiency of electron area detectors in electron microscopy," Ultramicroscopy, vol. 109, no. 9, pp. 1126-1143, Aug. 2009, doi: 10.1016/j.ultramic.2009.04.002.

[2] M. A. Herzik, M. Wu, and G. C. Lander, "Achieving better than $3 \AA$ A resolution by single particle cryo-EM at $200 \mathrm{keV,"} \mathrm{Nat} \mathrm{Methods,} \mathrm{vol.} \mathrm{14,} \mathrm{no.} \mathrm{11,} \mathrm{pp.} \mathrm{1075-1078,} \mathrm{Nov.} \mathrm{2017,} \mathrm{doi:} \mathrm{10.1038/nmeth.4461.}$ 
[3] R. Henderson, "The potential and limitations of neutrons, electrons and X-rays for atomic resolution microscopy of unstained biological molecules," Quarterly Reviews of Biophysics, vol. 28, no. 2, pp. 171-193, May 1995, doi: 10.1017/S003358350000305X.

[4] M. Khoshouei, M. Radjainia, W. Baumeister, and R. Danev, "Cryo-EM structure of haemoglobin at $3.2 \AA$ determined with the Volta phase plate," Nat Commun, vol. 8, no. 1, pp. 1-6, Jun. 2017, doi: $10.1038 /$ ncomms 16099 .

[5] M. A. Herzik, M. Wu, and G. C. Lander, "High-resolution structure determination of sub-100 kDa complexes using conventional cryo-EM," Nat Commun, vol. 10, no. 1, pp. 1-9, Mar. 2019, doi: 10.1038/s41467-019-08991-8.

[6] J. H. Mendez, A. Mehrani, P. Randolph, and S. Stagg, "Throughput and resolution with a nextgeneration direct electron detector," IUCrJ, vol. 6, no. 6, pp. 1007-1013, Nov. 2019, doi: $10.1107 /$ S2052252519012661.

[7] R. Henderson and G. McMullan, "Problems in obtaining perfect images by single-particle electron cryomicroscopy of biological structures in amorphous ice," Microscopy $(O x f)$, vol. 62, no. 1, pp. 43-50, Feb. 2013, doi: 10.1093/jmicro/dfs094.

[8] W. H. Massover, "Ultrastructure of ferritin and apoferritin: A review," Micron, vol. 24, no. 4, pp. 389-437, Jan. 1993, doi: 10.1016/0968-4328(93)90005-L.

[9] C. J. Russo and L. A. Passmore, "Ultrastable gold substrates for electron cryomicroscopy," Science, vol. 346, no. 6215, pp. 1377-1380, Dec. 2014, doi: 10.1126/science.1259530. 
https://doi.org/10.1017/S1431927620021182 Published online by Cambridge University Press 